\title{
Bridging the Gap Between Robotic Technology and Health Care
}

\author{
Adriano O. Andrade ${ }^{\mathrm{a}, *}$, Adriano A. Pereira ${ }^{\mathrm{a}}$, Steffen Walter $^{\mathrm{b}}$, Rosimary Almeida ${ }^{\mathrm{c}}$, Rui \\ Loureiro $^{\mathrm{d}}$, Diego Compagna ${ }^{\mathrm{e}}$, Peter J. Kyberd ${ }^{\mathrm{f}}$ \\ ${ }^{a}$ Biomedical Engineering Laboratory, Faculty of Electrical Engineering, Federal University of Uberlândia, \\ Uberlândia, Brazil \\ ${ }^{b}$ Medical Faculty, University of Ulm, Ulm, Germany \\ ${ }^{c}$ Biomedical Engineering Program, Alberto Luiz Coimbra Institute - Graduate School and Research in \\ Engineering (COPPE), Universidade Federal do Rio de Janeiro, Rio de Janeiro, Brazil \\ ${ }^{d}$ School of Science and Technology, Middlesex University, London, UK \\ ${ }^{e}$ Faculty of Social Sciences, University of Duisburg-Essen, Duisburg, Germany \\ ${ }^{f}$ Institute of Biomedical Engineering, University New Brunswick, Fredericton, Canada
}

\begin{abstract}
Although technology and computation power have become more and more present in our daily lives, we have yet to see the same tendency in robotics applied to health care. In this work we focused on the study of four distinct applications of robotic technology to health care, named Robotic Assisted Surgery, Robotics in Rehabilitation, Prosthetics and Companion Robotic Systems. We identified the main roadblocks that are limiting the progress of such applications by an extensive examination of recent reports. Based on the limitations of the practical use of current robotic technology for health care we proposed a general modularization approach for the conception and implementation of specific robotic devices. The main conclusions of this review are: (i) There is a clear need of the adaptation of robotic technology (closed loop) to the user, so that robotics can be widely accepted and used in the context of heath care; (ii) For all studied robotic technologies cost is still prohibitive and limits their wide use. The reduction of costs influences technology acceptability, thus innovation by using cheaper computer systems and sensors are relevant and should be taken into account in the implementation of robotic systems.
\end{abstract}

Keywords: robotics, health care, prosthetics, rehabilitation, companion robotic systems.

\section{Introduction}

In 1917 the term robot was for the first time used by the Czech dramatist Karel Capek [1]. In his popular scientific play RUR (Rossum's Universal Robots), Capek describes intelligent machines, that although created for serving humans, dominated the world and destroyed humanity [1].

\footnotetext{
${ }^{*}$ Corresponding author

Email address: aoandrade@feelt.ufu.br (Adriano O. Andrade)
} 
From that time on we have seen a great advance in technology for robot conception and implementation which seems to be governed by the law of Accelerating Returns [1] that suggests that the time interval between relevant events becomes shorter as time goes by, and the return, i.e, the value of technology, increases exponentially. The advance of technology is accompanied by the necessity of increasing computational resources, e.g., memory and computational speed, which is governed by Moore's law.

Although technology and computation power have become more and more present in our daily lives, we have yet to see the same tendency in robotics applied to health care. We argue that this is happening because robotic devices that have been developed are still far from obeying one of the most important principles of Cybernetics, which states that intelligent machines should adapt and react to their environment so that there is complete interaction between human and robots.

The aim of this review is to provide the reader with broad information about the application of robotic technology to health care. In this context we review four important applications, named Robotic Assisted Surgery, Robotics in Rehabilitation, Prosthetics and Companion Robotic Systems. We identify the main challenges these areas are facing and discuss possible solutions for them.

\section{Robotic Assisted Surgery (RAS)}

Any surgery is intrusive in nature, so there has been a strong motivation to the development of new technologies for reducing the complications of trauma related to operation by means of minimal invasive procedures through small orifices.

In this perspective, robotic assisted procedures can be seen as important tools that provide flexibility, stability and enhanced vision for professionals executing surgeries [2].

As any other new technology, RAS should be judged on its performance and costeffectiveness and not only on its technological persuasiveness.

\subsection{Existing systems}

There are many types of robotic technologies being developed in research laboratories and by companies all over the world. Camarillo et al. [3] suggest a classification of the use of robots in surgery based on the level of responsibility and involvement the robot has with the patient during a procedure. In this sense robots that are solely used as an automated positioning system, such as a patient-mounted robotic platform for CT-scan [4] are considered the most passive.

Some robotic positioning systems can be considered active because the way they interact with the patient. For instance, the CyberKnife Robotic Radiosurgery System (Accuray Sunnyvale, CA) is composed of a radiation delivery device, called a linear accelerator, which is mounted on a robotic arm. This system automatically registers the preoperative path by correlating real-time radiographic images with the preoperative CT images to locate and eliminate the tumor in the patient [3]. 
In 1994, the Food and Drug Administration (FDA) approved the use of AESOP (Auto-mated Endoscopic System for Optimal Positioning) - Computer Motion (Goleta,California). This system is a robotic endoscope holder, which is used to hold and position rigid endo-scopes during minimally invasive surgery. This robotic arm was designed to offer direct control over the laparoscope by means of a foot pedal and later on by voice control [5].

The concept of a master-slave telemanipulation system was developed in the early 1990s to overcome the issue of dexterity in complex procedures, and also with the goal of devel-oping telesurgery to operate on patients from remote places. Initially, Computer Motion developed the telemanipulator system ZEUS specifically for cardiac operations. Later on, ZEUS was also used for laparoscopic procedures in animal models to verify the feasibility and applicability of robotic systems in different surgical areas, including general surgery, gynecology, urology, and pediatric surgery $[5,6]$.

The NeuroMate (Integrated Surgical Systems) is the first United States Food and Drug Administration-approved, commercially available, image-guided, robotic-assisted system used for stereotactic procedures in neurosurgery [7]. The precision robot "Evolution 1" (U.R.S. Universal Robot Systems, Schwerin, Germany) is a neurosurgical tool that has 7 actuated axes; it is a universal instrument interface, a mobile pre-positioning system, including the control computer rack, and the touch operated graphical user interface [8].This system has been used for robot-assisted navigated endoscopic third ventriculostomies in patients with hydrocephalus related to aqueductal stenosis [8].

Hagn et al. [9] discuss the advantages and disadvantages of dedicated and versatile surgical robotic systems. The first type of system is dedicated for specific applications or diseases, whereas the latter can be adapted to a wide range of applications. In this context, the authors proposed the MiroSurge, which is a versatile robotic system that can be adapted to multiple surgical domains (e.g., visceral surgery and neurosurgery). The specialization of the system is obtained by the use of specialised instruments connected to drive units of the robot.

Currently, the Da Vinci Surgical System (Intuitive Surgical Inc, Sunnyvale, California, USA) is one of the most successful, general, commercial and studied surgical robot [10], which is used in clinical environments such as a hospital. This is a robotic master-slave system that consists of a remote console where the operating surgeon (master) directs the robotic surgical arms (slave) via a telerobotic videoscopic link, with the aim of facilitating certain surgical procedures. This system allows for enhanced stereoscopic and enlarged high definition imaging. It has the potential for tremor free precise movements and it uses intracorporeal articulated instruments with multiple degrees of freedom allowing partially overcoming the problem of the fulcrum effect seen with conventional laparoscopy using rigid instruments $[2,11]$. It also allows gearing down of the motions to make them more precise.

\subsection{Pros and cons}

Despite the growing of evidence for the successful use of RAS, most studies reported are case series from large centres, therefore there is lack of conclusive comparative studies. In the current literature mainly short-term follow-up outcome data are available [2, 10-15]. 
Table 1: Pooled estimate from a meta-analysis comparing Robotic-Assisted Radical Prostatectomy (RARP) and Laparoscopic Radical Prostatectomy (LRP) for outcome measures favours RARP.

\begin{tabular}{|c|c|c|c|c|}
\hline $\begin{array}{l}\text { Outcome } \\
\text { Measure }\end{array}$ & $\begin{array}{c}\begin{array}{c}\text { Number } \\
\text { of } \\
\text { Studies }\end{array}\end{array}$ & $\begin{array}{l}\text { Sample } \\
\text { Size }\end{array}$ & $\begin{array}{c}\text { Statistical } \\
\text { Heterogeneity } \\
\text { Measures } \\
\text { I }^{2}, \text { P-Value }\end{array}$ & $\begin{array}{l}\text { Pooled Estimate } \\
\qquad[95 \% \mathrm{Cl}]\end{array}$ \\
\hline $\begin{array}{l}\text { Operative time } \\
\text { (minutes) }\end{array}$ & 9 & 1,415 & $89.8 \%,<0.00001$ & $\begin{array}{l}\text { WMD -22.79 } \\
{[-44.36,-1.22]}\end{array}$ \\
\hline $\begin{array}{l}\text { Hospital stay } \\
\text { (days) }\end{array}$ & 7 & 1,235 & $76.2 \%, 0.0003$ & $\begin{array}{c}\text { WMD }-0.80 \\
{[-1.33,-0.27]}\end{array}$ \\
\hline Blood loss (mL) & 10 & 1,655 & $90.0 \%,<0.00001$ & $\begin{array}{c}\text { WMD -89.52 } \\
{[-157.54,-21.49]}\end{array}$ \\
\hline $\begin{array}{l}\text { Incidence of } \\
\text { transfusion }\end{array}$ & 7 & 1,820 & $0 \%, 0.83$ & $\begin{array}{c}\text { RR 0.54 } \\
{[0.31,0.94]}\end{array}$ \\
\hline
\end{tabular}

Legend: $\mathrm{CI}=$ confidence interval; $\mathrm{RR}=$ risk ratio; $\mathrm{WMD}=$ weighted mean difference. Pooled estimates are reported as WMD for continuous measures and as RR for dichotomous measures. Source: Extracted from the report of the Canadian Agency for Drugs and Technologies in Health [12].

Most of the available evidence on efficacy is for robotic assisted radical prostatectomy, which is also the largest current indication in the world [16]. There is evidence that perioperative blood loss is lower than with conventional techniques [2, 10-15] but, taking into account the most updated review [12], evidence for other expected advantages, such as reduced incontinence, reduced erectile dysfunction or shorter length of hospital stay, is less consistent and highly dependent on skill and experience of the surgical team (see Table 1).

The reviews [2, 10-15] highlighted that the main cost-drivers of the robot-assistance in surgery are the capital acquisition, around $€ 1.7$ million [12], and maintenance that is approximately $10 \%$ of the acquisition cost, followed by the high costs of limited re-usable surgical instruments.

\subsection{Future trends}

Several factors including establishing adequate access, two dimensional vision, decreased depth perception, restricted instrument maneuverability, decreased dexterity and dampened tactile feedback are all unique limitations that make robotic assisted surgery challenging for surgeons trained in traditional open approaches [17, 18].

Camberlin et al. [2] discuss some future development needed in robotic-assisted surgical systems: (i) Development of smaller, cheaper, faster, and safer devices with improved features such as haptic feedback; (ii) Improved instrumentation, such as smarter instruments with capabilities to do smart sensing, informing the surgeon about tissue oxygenation, blood flow, molecular information and even tumour margin information by intraoperative histology; (iii) Provision of additional help to the surgeon with anatomic overlays incorporating information from other sources, or even offering optical biopsy capabilities; (iv) Paradigm 
shift from intracorporeal tools attached to an extracorporeal device to entirely intracorporeal devices: intra-abdominal cameras and intracorporeal self propelled mobile robots could be used for microsurgery and other applications such as real-time intra-operative anatomy and histology, or for the delivery of new therapeutic techniques such as local phototherapy.

\subsection{Recommendations}

Over the past fifteen years we have had great advance in robotic surgery but its use is still limited to a few research centres and hospitals in the world. Researchers and engineers responsible for developing, implementing and assessing this technology should focus on critical issues that could bring it to the reality of patients. From the literature review it is possible to extract relevant messages that could guide researchers in the development and improvement of RAS:

- The evidence of the effectiveness, safety, costs and budget impact of robotic surgery should be evaluated in a broad range of procedures;

- New systems should minimize training costs and the learning curve of the user;

- More effort should be dedicated to the implementation of modular and versatile systems;

- Designs should prioritize the reusability of supplies.

\section{Robotics in Rehabilitation}

Rehabilitation incurs considerable costs to health care systems all over the world. Brain injuries, movement disorders and chronic pain affect hundreds of thousands of people worldwide and have a profound impact in their quality of life. Strokes are the third most common cause of death worldwide after heart disease and cancer (The Stroke Association, UK, 2008), and the most common cause of acquired physical disability. Tremor on the other hand, is one of the major causes of functional disability and the most common disorder in neurological practice, affecting mostly the elderly population [19-21]. Chronic neurological pain can worsen due to anxiety/stress and other factors. Not only does this affect the individual's mental state but also their physical state and rehabilitation outcome. This in turn poses diverse challenges to health services and rehabilitation centres as the individual experiences emotional discomfort in addition to psychological trauma and reduced mobility.

Although repetitive task-oriented movements are the single most important variable of motor skill acquisition [22, 23] and have a therapeutic gain [24, 25], the literature suggests that a patient recovering from neurological trauma must play an active role in the rehabilitation process if improvement is to occur. However, as motor learning is not a passive, imprinting process - requiring active problem-solving and experience - there needs to be enough capability for the patient to participate [26]. Consequently, providing patients recovering from a brain injury with meaningful goals (use of familiar objects with well-established sensory cues and known semantic properties) can potentially lead to better recovery. 


\subsection{Existing systems}

Physical rehabilitation of brain injuries and strokes is a time consuming and costly process. Over the past decade several studies have emerged looking at the use of highly sophisticated technologies, such as robotics and virtual reality to tap into the needs of clinicians and patients [27]. Although traditional physiotherapy coupled with machine mediated therapy can be beneficial in the recovery process, once patients are discharged from hospital it is difficult to exercise correctly the affected limb without the support from clinicians.

A number of robotic systems can be found in the literature. Limb rehabilitation can be classified into two categories, upper limb and lower limb rehabilitation, which can include sub-categories such as hand, wrist, elbow and shoulder rehabilitation.

A recent review on lower limb robotic rehabilitation [28] describes the most relevant available technologies. In general these systems can be grouped according to the rehabilitation principle they follow [28]: (i) treadmill gait trainers, (ii) foot-plate-based gait trainers, (iii) overground gait trainers, (iv) stationary gait trainers, (v) ankle rehabilitation systems (stationary systems and active foot orthoses). The description of a number of upper limb rehabilitation devices can be found in $[27,29,30]$.

\subsection{Pros and cons}

While such technologies can be a valuable tool to facilitate intensive movement practice in a motivating and engaging environment, success of therapy also depends on self-administered therapy beyond hospital stay. Despite all the technological evolution observed over the last decade, one of the main challenges is to how best use robotic technology to strengthen the physiotherapist's skills [31]. Robotic technologies are advanced tools and not a physiotherapist replacement, thus unlikely to assemble all the skills of a physiotherapist, but exceling at conducting simple repetitive intensive manual therapies. Hence, once clinical decisions are made, these can be considered and executed on the robot. This observation has been clearly demonstrated by some robotic therapy studies that matched the level of assistance and intensity between the intervention and control groups [32, 33]. In both studies com-parable improvements between the intervention and control groups were observed [32, 33]. So, what is the advantage of using robots, if intensive conventional physiotherapy delivered by human therapists can have similar functional gains on patients? For starters, given the pressures imposed on health systems and the lack of available therapists, it is unpractical during conventional therapy to maintain high levels of intensity, as it is possible with robots. One advantage of robotic therapy over conventional therapy is that robots allow therapists to take a step back from physically engaging in assisting the patient to perform repetitive movements. The robot can provide longer and more intensive repetitive assistance thus allowing therapists the opportunity to observe, make informed decisions on best course of action and manage more patients. This is perhaps the reason why most of the research to date concentrates on the principle of massed practice. Robotic therapy is appealing because it can deliver complex therapies that would be too difficult for therapists to do, for instance 
provision of precise repeatable force and haptic feedback coupled with interesting and motivating visual feedback andor the ability to augment movement errors to help correct a movement pattern [34].

\subsection{Future trends}

A recent review $[27,35]$ has concluded that despite mounting evidence suggesting robotic therapies are not more likely to improve patients' activities of the daily living than any other therapy, it has shown great capacity to improve patients' motor function and core strength $[27,35-37]$. The consensus exists that equal importance should be placed by clinicians and researchers working in the field in establishing guidelines on the study design and assessment and on pushing for more efficient, safer and inexpensive technologies. To this effect, the International Consortium on Rehabilitation Robotics and the COST European Network on Neurorehabilitation are undertaking a cross-disciplinary basic and applied research coordinated efforts to aid the development of new, efficient and patient-tailored robot-assisted therapies. Through the provision of structured overviews relating to current and emerging robot-assisted therapies to clinicians and therapists, the group expects this will increase the availability of effective and standardised clinical practice across Europe. It is expected that this initiative will pave the way for an international effort to increase the numbers on robotic trials by considering nonclinical sensitive measures with a common treatment and measurement protocol independent from the robot platform.

A hospital or clinical environment might use devices able to retrain a variety of movements over a large percentage of the normal range of human movements. However, an emphasis should be placed on allowing the implementation of therapies resembling activities of the daily living such as, picking up a book after reading at a table top and placing it on a bookshelf. Loureiro and Smith [38] for example propose a multimodal robotic system that encourages arm and hand movements in addition to stabilisation of the trunk while moving from a sitting to a standing posture or maintaining a standing pose. The ROBIN system (see Fig. 1) was designed to deliver therapies supporting activities of the daily living whilst combining retraining of simple reach and grasp movement (while seating/standing), which could have a meaningful transfer to everyday functional gains and higher functional independence. The ROBIN system is based on previous work with the Gentle/G system [39] and following recent results from a reach and grasp study [40] it is being used to investigate the effects of dynamic gravity compensation in reach and grasp movements during standing or sitting tasks. The authors anticipate that the ROBIN system will lead to the development of hybrid control methodologies that estimate the internal state of the patient through a multimodal approach combining several sensor information. Emerging evidence from func-tional neuro-imaging suggests that task-oriented sensorimotor participation through daily training of the arm and hand can positively influence stroke recovery [41-43]. Optimiz-ing robotic rehabilitation on functional outcome should take into account exposures of the nervous system to real-life activities during therlapgre is a need for more effective tools so that neuro-therapies can be moved away from the therapy gymnasium and into the person's home. These tools have the potential to make a large impact on the recovery of people following their stroke, as therapy will be available 

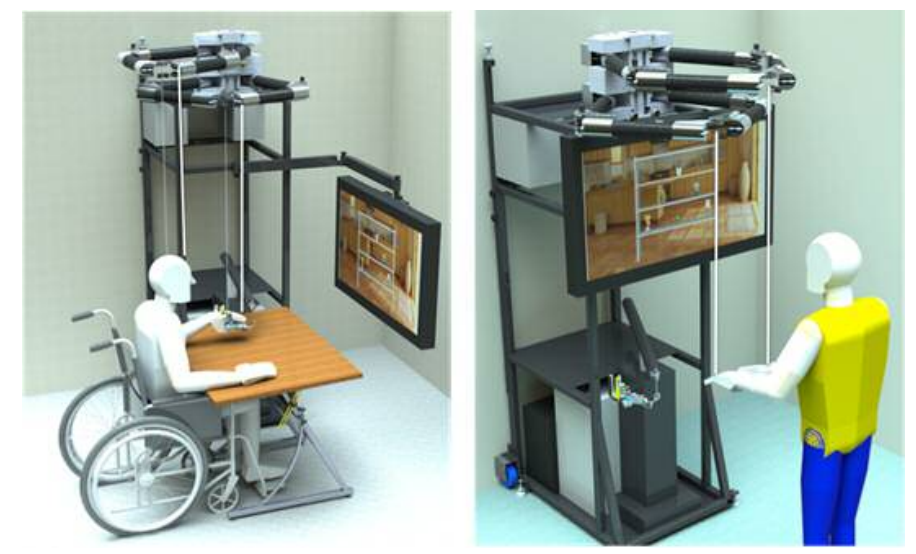

Figure 1: Illustration of the ROBIN system. (left) used while seating, (right) used while standing (reprinted with permission: [38]).

on demand at the convenience and in a familiar environment to the patient. As part of this therapy process we can exploit the dual nature of robotic devices to both assist and measure movement. Rehabilitation robots that are always available provide unbiased and consistent therapies coupled with intrinsic measurements can quantify and possibly model therapy progress. Robotic assistance is particularly useful where patients lack control of movement and less complex systems based on sensor feedback might be more useful in the home when dexterity control still lacks and there is a need to motivate the patient to engage in the therapy. Although several groups are active in the field of rehabilitation recent developments have concentrated in implementing new devices and systems that will assist in the recovery of the upper limb robotics [44-47].

With the emergence of low-cost gaming consoles such as the Nintendo Wii and the Microsoft Xbox Kinect, new opportunities arise for home-therapy paradigms centred on social interactions and values, which could reduce the sense of isolation and other depression related complications. New approaches combining telerehabilitation concepts with collaborative play [48], and simple robotic sonic interaction [49] have the potential to increase engagement and participation of individuals in remote and localized group therapy.

New approaches combining telerehabilitation concepts with collaborative play [48], and simple robotic sonic interaction [49] have the potential to increase engagement and participation of individuals in remote and localized group therapy. A recent study combining exploration of a painting aided by haptic and sound cues, concluded that group interaction resulted in increased engagement with the interactive installation and increased execution of movements [40, 50]. This work showed the potential for such interventions for development of analytical skills, imagination, promotion of spatial skills realisation and enhancement of touch/hearing sensory channels. Although such approaches might be of value to neurorehabilitation, such concepts of augmented artefact installations with technology need to be carefully designed to promote social integration and potential use in public spaces.

While clinical scales can help us to examine the impact in the neuro-recovery process, their coarse nature requires extensive and time-consuming trials, and on top of that they fail to show us details important for optimizing therapy. Alternative, robot-based scales 
offer the potential benefit of new finer measurements-and deeper insight into the process of recovery from neurological injury [51].

\subsection{Recommendations}

One of the main roadblocks to progress in the field is the need for evidence on the effec-tiveness of rehabilitation robots. The cost of rehabilitation robots is still high then compared to drug-based and humanbased therapies, which in turn makes wide-scale evaluation of such therapies difficult. Often studies have been limited to pilot evaluations, typically of less than 50 participants, to demonstrate the device's basic working principles [52].

A systematic review on the clinical effect of robot-aided therapy [36] have concluded that although substantial improvements in short-term outcomes have been reported, upper limb robotic therapy fails to transfer such gains into higher level functional independence. A more recent Cochrane report [37] reviewing electromechanical-assisted gait training concluded that while evidence emerges showing recovery improvements of independent walking in people following a stroke, the importance of this type of devices and how it should be used in clinical practice is still unclear. Suggesting that further research should address what frequency or duration of walking training might be most effective and consequently how long the benefit can last.

The lack of conclusive evidence generated thus far has an impact on the acceptance of robotic therapies in clinical practice. Clinical outcomes reported in the literature vary [27] and might be ascribed to variations on patient characteristics, exposure to therapies and intensity. This in turn has implications to the study design and prompts for careful interpretation of the data.

\section{Prosthetics}

Prosthetics is the oldest of the technological rehabilitation sciences and for as long as people have been surviving the trauma of lost limbs, there have been persons motivated to replace some of the functions of those limbs by building prostheses. It is not uncommon that these innovators have been the persons with amputations themselves.

\subsection{Existing systems}

A possible classification of artificial limbs may consider the way the prosthesis is powered for generating the required movements. Based on this criterion, prosthetic devices can be subdivided into four classes: Passive Prostheses, Body-Powered Prostheses, Externally-Powered Prostheses and Hybrid Prostheses [53].

Passive Prostheses are completely passive, i.e., they can only be moved and placed in desired positions through external forces, and often are used for cosmetic reasons. The use of these types of devices may have a positive psychological effect on the individual by increasing self-esteem [53]. 
Body-Powered Prostheses are operated so that the force of movement of a body part reflects the execution of the movement of the prosthesis. Movements of the shoulder and other parts of the body can be used for controlling the artificial limb [53].

Externally-Powered Prostheses are those which are energized by some external source of energy, e.g., batteries. These prostheses are usually controlled by the movement of remaining natural structures or by electrical muscle activity provided by a chosen muscle group [53].

Hybrid Prostheses are those in which the control functions of the limb (e.g., electrical muscle activity) can be combined with a control system via harnesses for the functions of the elbow. This type of prosthesis is known as hybrid prosthesis because it combines two types of control in the same prosthesis [53].

\subsection{Pros and cons}

In this section the distinct types of existing prosthetic devices are discussed in terms of their control, which can be considered one of the most interesting challenges related to prosthetics. Ideally, a prosthetic limb should be controlled without any effort from the user, similar to the subconscious control of a natural limb.

There are only two practical sources of power for the prosthetic limb: body powered, in which the motion is provided by harnessing the motion of some other body part and mechanically linking that part to the prosthesis; or electrically powered using rotary electric motors (permanent magnet or brushless). Other sources have been suggested [54-56], but so far they have not been used beyond the laboratory. The reasons are generally practical: to recharge a gas cylinder for a pneumatic arm is possible, but complex to do in a domestic environment, or to create hydraulic systems that can provide enough force without being too heavy. However electric motors are ill suited to provide the stop-start motion required of a prosthetic limb. Hence the idea from Schultz et al. to use an electric motor in a hand to provide the pressure for an hydraulic system [54].

The first prostheses were generally passive devices that relied on intact parts of the body for their positioning and controlling. In the late 19th century the first body-powered prostheses were developed. This extremely successful design allowed the user to control the device such that the movement of a part of the body was reflected in the movements of parts of the prosthesis [53].

Despite some modifications, currently, this design remains basically the same and the control mechanism is the most popular among users. The reasons for this success are not well established, but according to Doeringer and Hogan [57] some of the key factors are: it results in a relatively inexpensive prosthesis; the final prosthesis is not too heavy; after training, the user begins to use the prosthesis as a natural extension of his body, having, for example, the notion of weight and size of the prosthetic limb.

Kruit and Cool [58] described the main drawbacks of the mechanism described above: the mechanism of harnesses used to propagate the movements of the body is usually uncom-fortable; the movement of the prosthesis requires significantly large forces; the number of control inputs is limited and thus the number of degrees of freedom of the prosthesis is also limited. An alternative to the body-powered control is to employ the myoelectric control, which uses the electrical activity of muscle contraction as a controlling signal for prostheses. 
While switches and levers are other means to control the limbs, it is generally myoelectric signals that are used to instruct and control powered prosthetic arms [59]. It is also being investigated as the means to control newer powered lower limb system.

Electromyographic signals are those generated by muscle as they contract. The resulting signal is noisy and prone to interference, both electrical and mechanical. While much can be done to ameliorate these extraneous signals, what is really being sought is to measure the intent of the user, which would require connections to the nerves or cortex of the brain. Progress in this direction is being made [60], but it is uncertain how popular such invasive surgery would be, when it has been developed enough to be useful. For example, it is a salutary lesson that the take up for neural stimulation of muscles for persons with paralysis is low, despite having some very compelling advantages over external stimulation. This group of patients has a great deal more to gain from the implants that a prosthesis user would, and yet few are interested. It is probable that for surgical implants to become more popular to either group, the results of the implant would need to be seen to give users a much greater advantage than they currently do. So far, the number of channels for connection in either direction is very limited and so control would have to be limited also.

The idea of myoelectric control is not new and came about in 1948 [61]. Electromyog-raphy has established as the most common biopotential employed for controlling artificial limbs, however over the past 15 years there is ongoing research seeking other forms of control based on more natural controlling strategies, such as those that employ brain or neuronal activity together with sensory feedback [56, 62-64].

In the past, myoelectric prostheses employed a type of controlling called "two-site twostates", from which a pair of electrodes is placed on two distinct muscles. The contraction of one of these muscles produces the opening of the hand. The antagonist muscle is used in the same way to control the closing of the hand. As pointed out by Scott and Parker [61], this approach works in a manner analogous to the human body, i.e., two antagonistic muscles (or group of muscles) control the movement of a joint.

However, as patients must learn to generate independent contractions of the muscles, which require a high degree of concentration, the training can be lengthy, requiring a lot of mental effort. There are also some situations in which it is not possible to find two available groups of muscles, and also the need of controlling more than one joint. For these situations other controlling approaches have been developed. For instance, the "one-site three-states", from which a little contraction of muscles produces the closing of the hand, a strong contraction open it, and the lack muscle activity stops the hand.

Currently there are a number of methods using proportional control based on the electri-cal muscle activity to control the speed, torque and position of prosthetic joints. However, due to the random nature of the myoelectric signal, errors and inaccuracies occur for various reasons [65]. Moreover, the learning process involved in the generation of myoelectric pat-terns must be learned by the user, and this is a task which requires concentration, regular training and a great amount of physical effort, making the translation of myoelectric activity into commands for a prosthetic limb a challenge [66]. 


\subsection{Future trends}

The designs of the prostheses and the functions they replace are as varied as the persons who have the loss. While a generic desire to replace what is missing is often foremost in the minds of the person with a recent loss, work by McLaughlin and Desmond of Trinity College Dublin [67], have shown that the persons' desires for their prosthesis change with time. Anecdotally, there is a complex relationship between desire for a like-life replacement and the level of loss. So that the person with a digital loss may wish something so natural at to be unobtainable, while those with a high level of loss at the shoulder, may wish merely to manipulate objects effectively and careless for appearance. This creates a need for a wide range of different solutions using knowledge from materials science to neurology. Since every potential user is an individual, the device must be customised to the user's loss, needs and cognitive ability to use the limb. Surveys of different patients therefore show these different requirements [68-70]. For this paper future trends will be discussed taking into account the following relevant requirements: Attachment, Appearance, Actuation and Control.

\subsubsection{Attachment}

Conventional prosthetic attachment has been to make a socket to fit around the residual limb. It needs to fit closely enough to allow easy transfer of forces to and from the prosthesis to the world for manipulation. It cannot be too tight to restrict blood flow and so for some persons, depending on the level and form of the loss, additional straps may be needed to hold the limb in place. Historically, the socket was made of metal, and more recently plastics. Now rubber roll-on sleeves spread the load evenly and have fewer pressure points between the limb and the socket. However, all of these solutions create problems with cooling. Animals use their skin to radiate heat and so help to control their internal temperature. The loss of a limb creates a loss of a significant percentage of the radiative area of the body [71]. Adding other materials on the residuum reduces the area still further, a rubber roll-on liner being a particular barrier to heat loss. There are now groups looking at new materials to add to the rubbers in liners to increase heat transfer [71]. However there has been little work looking at active heat transfer for the limb absent population.

One form of attachment that can circumvent the problem of a socket which is rigid, hard and likely to impede joint range of motion, is that of attaching the prosthesis directly to the bone. First demonstrated by Per-Ingvar Brannemark [72] attachment by Osseointegration is a standard technique for teeth and there are several million teeth world wide attached this way. There are other methods with variations on the theme, but so far no other team has such extensive experience [73].

An advantage of Osseointegration is that the person can feel through the bone. This so called "Osseoperception" has been observed to change the size and shape of the amputee's phantom image of their residuum. The person with a loss in the leg no longer is floating in mid air, but connects to the ground. Osseointegration's drawback is the same as all surgical interventions, there are many persons with a limb absence that will not welcome such an invasion. 


\subsubsection{Appearance}

The natural, immediate, response to the loss of a bodily part is often to want something that closely resembles the missing part. However as time goes on this tendency can change. When the person wants function they will often opt for less anthropomorphic and more functional, whether this is a carbon fibre c-shaped blade for sprinting, or a hook for handling tools. A person with an amputation may adopt different prostheses for different specific tasks (each user is unique with their requirements and needs), but generally the interest is in a device with a pleasing appearance, not necessarily a human like one. Masahiro Mori proposed the Uncanny Valley as a manifestation of the acceptability of a human-like object [74]. Based on Freud's ideas of The Uncanny, this idea suggests that the more lifelike an object or robot is the more acceptable it is until it reaches a point where it becomes "spooky", when it becomes unacceptable. Beyond this, once it is very close to life-like (such as a living being) it becomes acceptable again, creating the valley in a plot of acceptability against appearance. Mori suggested that prostheses are on the acceptable side of the rift. Anecdotal evidence suggests this is untrue. Prosthetic users ask for greater and greater realism in their prosthesis, but at some point they will reject it as "too spooky", hence the device has fallen in the valley. Anecdotally, it can be seen that the new high tech limbs attract some users who wish to show off their limbs and not hide them in cosmetic coverings. What is clear is that any covering for a prosthesis, designed to protect the mechanics of the device from dirt and ingress of moisture, needs to be resilient, resistant to tearing, while also flexible not impeding the motion of the limb. So far, the rubbers developed for this task fall far short of the target of fulfilling all of the above and being cheap enough to be practical.

\subsubsection{Actuation}

To ensure that the widest range of people can use a limb it must retain all its drives within the envelope of the missing joint. So a hand must have all the drives for the fingers within the hand $[75,76]$. To do otherwise would reduce a small market to an even smaller one. As the amputation level moves up the arm the numbers of persons reduces considerably [77], hence to restrict the market to those persons would be ineffective. This is a tighter requirement than Natural Selection created for the limb, where the muscles for the hand are in the forearm, and some of those for the shoulder are in the chest. Thus the design of the limb might well reduce the number of degrees of freedom to fewer than those lost. For example, even the most sophisticated prosthetic wrist used by human subjects in the field does not incorporate radial/ulnar deviation [78].

\subsubsection{Control}

This is tied up very closely with the means to actuate the device, since a body powered limb uses the motions of the body to drive the prosthesis and the feedback sense of how the device is moving allows for modification of its motions, hence its control.

One of the ways that EMG control of the limb is being enhanced is to train a computer to recognise the patterns of muscle signals across the residual limb [79, 80] as an arm is moved. One flaw in the ability to detect signals is that the amputation reduces the number of possible sites to derive a control signal. Kuiken conceived of the idea of Targeted Muscle 
Reinnervation (TMR). This is based on the observation that if a nerve end is placed in a muscle it will remake connections. While for reinnervation of intact limbs the low rate of reattachment is a disappointment, Kuiken realised if he denervated a muscle that was not being used (for example the pectoralis in someone with a loss at the shoulder), then divided the muscle and placed a nerve from a muscle that was lower down the arm (such as the radial nerve), then some control sites would be created. Effectively the muscle is amplifying the nervous signal $[81,82]$. TMR is still an invasive technique and requires much training to be useful [83].

Pattern recognition in general, is an appealing idea, but so far there have been no genuine clinical demonstrations of the technique reported, although this is likely to change within the next year or so. An important advance in this field is Prosthesis Guided Training (PGT)[84], which allows the user to retrain the system at any time by having the device go through preset motions and then the user copies those motions. This gets round any idea that the system is fixed, it can be adapted to the changing of the user due to experience or fatigue. If Pattern Recognition has a practical future, it will only be in conjunction with some form of user triggered retraining.

One problem with most of the control formats in use is that they have been pragmatically developed with little reference to the way that the natural solutions have evolved. An example is that the motion of fingers and thumb are different to each other [85], but when the action of a user was measured using a hand where the fingers and thumb moved in the same way, the motions of the user were such to reproduce (or get closer to) the natural motions [86]. The new frontier is to understand more fully how control is achieved in the natural hand or leg and adapt the training and use of the devices to better match the way we use the prostheses [87].

\subsection{Recommendations}

The information and discussion presented in this section suggest that for the advance-ment of prosthetics and its acceptability by users a number of barriers should be overcome:

- The mental effort required for controlling a prosthetic device is still high, requiring training and concentration. The current available technology may not be accessible to individuals suffering from even mild cognitive disability;

- The use of information from nerves and also the brain for controlling an artificial limb is still a promise. Future research should try to clearly show the clinical usefulness of these methods;

- The types of materials used for attachment of the prosthesis to the body should promote natural heat change;

- The costs of an artificial limb are considerably high, thus the use of cheaper materials in its design is essential for its acceptance and popularization; 
- New actuators based on smart materials should be investigated in order to overcome known problems related to electric motors and hydraulic systems;

- Although a number of studies show the success of Pattern Recognition to the control of prostheses (specially upper limb devices) there is a lack of clinical evidence showing the actual success of the method;

- More understanding of how control is achieved in the natural limbs is necessary so that customized training protocols can be devised to the individual.

\section{Companion Robotic Systems for Health Care}

To improve and to ensure the quality of life for the elderly and disabled is an essential task of our society. The rising number of elderly with respect to the overall population and corresponding decrease of available care personnel calls for new health care concepts and solutions. By taking over repetitive and physically demanding tasks, advanced technology can further support the personnel of eldercare facilities in their daily work and thus allow them to focus their work on actual care tasks.

In this context, Companion Robotic Systems for Health Care (CRSHC), which bind together science, engineering, society and health care are relevant and recent robotic technology capable of offering additional psychophysical aid to individuals. Currently is its possible to find a number of studies focused on the development of CRSHC. Most of them aim to develop methods and technologies for the construction of cognitive robots, able to evolve and grow their capacities in close interaction with humans in an open-ended fashion. A practical application of a companion robot could be the cognitive stimulation and therapy management for the elderly by means of a robotic companion working collaboratively with a smart home environment.

Due to the sensibility of the field as a morally charged context and its characterization as a highly human driven 'relational' work sector the development should be guided by participatory methods - i.e. the development should be driven by user's needs.

Adopting the appropriate participatory methods is crucial to avoid the risk of first enhancing/ improving the care-giving and its setting by strengthening key practices and then fragmenting/ reducing it to a series of interaction loops and tasks that could be described as interaction algorithms and by that able to be carried out by CRSHC.

\subsection{The role of Requirements Engineering in the conception of CRSHC}

Requirements engineering is a necessary condition for an innovation process guided by the actual needs of the potential users. The idea is based on a number of works that emphasize the importance of potential users in the context of innovative technologies [88, 89]. Users play a crucial role in the process of the diffusion of an innovation [90]. Therefore they should participate already in the early phase of development until a stable shape of a technological system is reached [91]. Requirements engineering provides a functional contribution to the development of innovative technologies. This implies that this method raises the acceptance 
needed to gather additional meaningful experience with the performance of technology in daily practice.

Despite implicit or even explicit claims of the superiority of robotic systems for health care, when compared to more traditional methods, the clear advantage of these systems are currently unproven and are highly dependent on the skills of the users, therefore, the success of such technologies are still heavily dependent on adequate training and experience.

Based on the limitations of the practical use of current robotic technology for health care we proposed a general modularization approach for the conception and implementation of specific robotic devices. The system should have an individual adaptation module and many specific submodules related for the following aspects: disease, diagnostics, needs, cognition, dialog preferences, emotion and pain.

\section{Acknowledgment}

The authors would like to thank the Brazilian agencies CNPq, CAPES and FAPEMIG for supporting research carried out at the Biomedical Engineering Laboratory of the Faculty of Electrical Engineering, Federal University of Uberlândia. The Transregional Collaborative Research Centre SFB/TRR 62 "Companion-Technology for Cognitive Technical Systems" funded by the German Research Foundation (DFG).

\section{References}

[1] R. Kurzweil, The age of spiritual machines: how we will live, work and think in the new age of intelligent machines, Orion Business, London, 1999.

[2] C. Camberlin, A. Senn, M. Leys, C. De Laet, Robot-assisted surgery: health technology assessment health services research (hsr), Tech. Rep. 104C (D/2009/10.273/09), Belgian Health Care Knowledge Centre (KCE), Brussels, Belgium (February 2009).

[3] D. B. Camarillo, T. M. Krummel, J. Salisbury, J. K., Robotic technology in surgery: past, present, and future, Am J Surg 188 (4A Suppl) (2004) 2S-15S.

[4] B. Maurin, B. Bayle, O. Piccin, J. Gangloff, M. de Mathelin, C. Doignon, P. Zanne, A. Gangi, A patient-mounted robotic platform for ct-scan guided procedures, IEEE Trans Biomed Eng 55 (10) (2008) 2417-25.

[5] J. Marescaux, F. Rubino, The zeus robotic system: experimental and clinical applications, Surg Clin North Am 83 (6) (2003) 1305-15, vii-viii.

[6] M. Eto, S. Naito, Robotic manipulators in cardiac surgery: the computer-assisted surgical system zeus, Minimally Invasive Therapy and Allied Technologies 10 (6) (2001) 275-81.

[7] N. Nathoo, M. C. Cavusoglu, M. A. Vogelbaum, G. H. Barnett, In touch with robotics: Neurosurgery for the future, Neurosurgery 56 (3) (2005) 421-431.

[8] M. Zimmermann, R. Krishnan, A. Raabe, V. Seifert, Robot-assisted navigated endoscopic ventriculostomy: implementation of a new technology and first clinical results, Acta Neurochirurgica 146 (7) (2004) 697-704.

[9] U. Hagn, R. Konietschke, A. Tobergte, M. Nickl, S. Jorg, B. Kubler, G. Passig, M. Groger, F. Frohlich, U. Seibold, L. Le-Tien, A. Albu-Schaffer, A. Nothhelfer, F. Hacker, M. Grebenstein, G. Hirzinger, Dlr mirosurge: a versatile system for research in endoscopic telesurgery, Int J Comput Assist Radiol Surg 5 (2) (2010) 183-93.

[10] R. Tooher, C. Pham, The da vinci surgical robotics system: Technology overview, Tech. Rep. ASERNIP-S Report No 45, Australian Safety and Efficacy Register of New Interventional ProceduresSurgical, Adelaide, South Australia (July 2004). 
[11] P. Thavaneswaran, Robotic-assisted surgery for urological, cardiac and gynaecological procedures, Tech. Rep. ASERNIP-S Report No 75, Australian Safety and Efficacy Register of New Interventional Procedures-Surgical, Adelaide, South Australia (May 2009).

[12] C. Ho, E. Tsakonas, K. Tran, K. Cimon, M. Severn, M. Mierzwinski-Urban, J. Corcos, S. Pautler, Robot - assisted surgery compared with open surgery and laparoscopic surgery: Clinical effectiveness and economic analyses, Tech. Rep. CADTH No 137, Canadian Agency for Drugs and Technologies in Health, Ottawa, Canada (September 2011).

[13] S. M. Martínez, M. R. Goyanes, M. G. Rodríguez, J. A. B. Amaro, Efectividad, seguridad e indicaciones del equipo quirúrgico Da Vinci, plan de calidad para el sistema nacional de salud del ministerio de sanidad y política social Edition, Informes de Evaluación de Tecnologías Sanitarias, Agencia Laín Entralgo, Madri, Spain, 2009.

[14] A. L. Méndez, R. V. Portero, Cirugía robótica mediante el sistema de telemanipulación robótica da Vinci en la prostatectomía, Informes de Evaluación de Tecnologías Sanitarias, Agencia de Evaluación de Tecnologías Sanitarias de Andalucía, Sevilla, Spain, 2010.

[15] M. A. Secretariat., Robotic-assisted minimally invasive surgery for gynecologic and urologic oncology: an evidence-based analysis, Ontario Health Technology Assessment Series [Internet] 10 (27) (2010) $1-118$.

[16] C. Ho, E. Tsakonas, K. Tran, K. Cimon, M. Severn, M. Mierzwinski-Urban, J. Corcos, Pautler, Robot-assisted surgery compared with open surgery and laparoscopic surgery, CADTH Technology Overviews 2 (2) (2012) e2203.

[17] S. H. Baik, Robot Surgery, InTech, Rijeka, Croatia, 2010.

[18] D. M. Herron, M. Marohn, S.-M. R. S. Consensus, A consensus document on robotic surgery, Surgical Endoscopy and Other Interventional Techniques 22 (2) (2008) 313-325.

[19] M. Manto, M. Topping, M. Soede, J. Sanchez-Lacuesta, W. Harwin, J. Pons, J. Williams, S. Skaarup, L. Normie, Dynamically responsive intervention for tremor suppression, IEEE Engineering in Medicine and Biology Magazine 22 (3) (2003) 120-132.

[20] M. F. Almeida, G. L. Cavalheiro, A. A. Pereira, A. O. Andrade, Investigation of age-related changes in physiological kinetic tremor, Annals of Biomedical Engineering 38 (11) (2010) 3423-39.

[21] P. H. Mansur, L. K. Cury, A. O. Andrade, A. A. Pereira, G. A. Miotto, A. B. Soares, E. L. Naves, A review on techniques for tremor recording and quantification, Critical Reviews in Biomedical Engineering 35 (5) (2007) 343-62.

[22] R. Schmidt, Motor learning principles for physical therapy, Foundation for Physical Therapy, Alexandria, VA, 1991, pp. 49-63.

[23] C. Winstein, Knowledge of results and motor learning - implications for physical therapy, Physical Therapy 71 (1991) 140-149.

[24] C. Butefisch, H. Hummelsheim, P. Denzler, K. H. Mauritz, Repetitive training of isolated movements improves the outcome of motor rehabilitation of the centrally paretic hand, Journal of the Neurological Sciences 130 (1) (1995) 59-68.

[25] S. Hesse, C. Bertelt, M. Jahnke, A. Schaffrin, P. Baake, M. Malezic, K. Mauritz, Treadmill training with partial body weight support compared with physiotherapy in non- ambulatory hemiparetic patients, Archives of Physical Medicine and Rehabilitation 75 (10) (1995) 1087-93.

[26] R. Held, A. Hein, Movement-produced stimulation in the development of visually guided behavior, Journal of Comparative and Physiological Psychology 56 (5) (1963) 872-876.

[27] R. C. V. Loureiro, W. S. Harwin, K. Nagai, M. Johnson, Advances in upper limb stroke rehabilitation: a technology push, Medical \& Biological Engineering \& Computing 49 (1) (2011) 1103-1118.

[28] I. Díaz, J. J. Gil, E. Sánchez, Lower-limb robotic rehabilitation: Literature review and challenges, Journal of Robotics 2011 (2011) 1-11.

[29] S. Masiero, M. Armani, G. Rosati, Upper-limb robot-assisted therapy in rehabilitation of acute stroke patients: focused review and results of new randomized controlled trial, Journal of rehabilitation research and development 48 (4) (2011) 355-66.

[30] A. E. Q. van Delden, C. E. Peper, G. Kwakkel, P. J. Beek, A systematic review of bilateral upper 
limb training devices for poststroke rehabilitation, Stroke Research and Treatment 2012 (2012) 1-17.

[31] W. S. Harwin, J. L. Patton, V. R. Edgerton, Challenges and opportunities for robot-mediated neurorehabilitation, Proceedings of the IEEE 94 (9) (2006) 1717-1726.

[32] L. E. Kahn, M. L. Zygman, W. Z. Rymer, D. J. Reinkensmeyer, Effect of robot-assisted and unassisted exercise on functional reaching in chronic hemiparesis, in: Proceedings of the 23rd Annual International Conference of the IEEE, Vol. 2, pp. 1344-1347.

[33] A. C. Lo, P. D. Guarino, L. G. Richards, J. K. Haselkorn, G. F. Wittenberg, D. G. Federman, R. J. Ringer, T. H. Wagner, H. I. Krebs, B. T. Volpe, J. Bever, C. T., D. M. Bravata, P. W. Duncan, B. H. Corn, A. D. Maffucci, S. E. Nadeau, S. S. Conroy, J. M. Powell, G. D. Huang, P. Peduzzi, Robot-assisted therapy for long-term upper-limb impairment after stroke, The New England journal of medicine 362 (19) (2010) 1772-83.

[34] J. L. Patton, M. E. Stoykov, M. Kovic, F. A. Mussa-Ivaldi, Evaluation of robotic training forces that either enhance or reduce error in chronic hemiparetic stroke survivors, Exp Brain Res 168 (3) (2006) 368-83.

[35] S. Carda, M. Invernizzi, A. Baricich, C. Comi, A. Croquelois, C. Cisari, Robotic gait training is not superior to conventional treadmill training in parkinson disease: A single-blind randomized controlled trial, Neurorehabilitation and Neural Repair 26 (9) (2012) 1027-1034.

[36] G. B. Prange, M. J. Jannink, C. G. Groothuis-Oudshoorn, H. J. Hermens, M. J. Ijzerman, Systematic review of the effect of robot-aided therapy on recovery of the hemiparetic arm after stroke, J Rehabil Res Dev 43 (2) (2006) 171-84.

[37] J. Mehrholz, B. Elsner, C. Werner, J. Kugler, M. Pohl, Electromechanical-assisted training for walking after stroke, Cochrane Database Syst Rev 7 (2013) CD006185.

[38] R. Loureiro, T. Smith, Design of the robin system: whole-arm multi-modal sensorimotor environment for the rehabilitation of brain injuries while sitting or standing, in: Proceedings of the IEEE International Conference on Rehabilitation Robotics (ICORR), IEEE, 2011, pp. 1-6.

[39] R. C. V. Loureiro, W. S. Harwin, Reach \& grasp therapy: Design and control of a 9-dof robotic neurorehabilitation system, in: IEEE 10th International Conference on Rehabilitation Robotics (ICORR 2007), pp. 757-763.

[40] R. U. I. Loureiro, W. Harwin, R. Lamperd, C. Collin, Evaluation of reach and grasp robot-assisted therapy suggests similar functional recovery patterns on proximal and distal arm segments in sub-acute hemiplegia, IEEE Transactions on Neural Systems and Rehabilitation Engineering (2013) 1-1.

[41] J. Liepert, I. Uhde, S. Graf, O. Leidner, C. Weiller, Motor cortex plasticity during forced-use therapy in stroke patients: a preliminary study, J Neurol 248 (4) (2001) 315-21.

[42] S. H. Jang, Y. H. Kim, S. H. Cho, J. H. Lee, J. W. Park, Y. H. Kwon, Cortical reorganization induced by task-oriented training in chronic hemiplegic stroke patients, Neuroreport 14 (1) (2003) 137-41.

[43] G. F. Wittenberg, R. Chen, K. Ishii, K. O. Bushara, S. Eckloff, E. Croarkin, E. Taub, L. H. Gerber, M. Hallett, L. G. Cohen, Constraint-induced therapy in stroke: magnetic-stimulation motor maps and cerebral activation, Neurorehabil Neural Repair 17 (1) (2003) 48-57.

[44] S. E. Fasoli, H. I. Krebs, J. Stein, W. R. Frontera, R. Hughes, N. Hogan, Robotic therapy for chronic motor impairments after stroke: Follow-up results, Archives of Physical Medicine and Rehabilitation 85 (7) (2004) 1106-1111.

[45] P. S. Lum, C. G. Burgar, M. Van Der Loos, P. C. Shor, M. Majmundar, R. Yap, Mime robotic device for upper-limb neurorehabilitation in subacute stroke subjects: A follow-up study, Journal of Rehabilitation Research and Development 43 (5) (2006) 631-642.

[46] D. Reinkensmeyer, S. Housman, If I can't do it once, why do it a hundred times?: Connecting volition to movement success in a virtual environment motivates people to exercise the arm after stroke, in: Proceedings of the IEEE Virtual Rehabilitation, Venice, Italy, 2007, pp. 44-48.

[47] S. Masiero, A. Celia, G. Rosati, M. Armani, Robotic-assisted rehabilitation of the upper limb after acute stroke, Archives of Physical Medicine and Rehabilitation 88 (2) (2007) 142-149.

[48] M. J. Johnson, R. C. V. Loureiro, W. S. Harwin, Collaborative tele-rehabilitation and robot-mediated therapy for stroke rehabilitation at home or clinic, Intelligent Service Robotics 1 (2) (2008) 109-121. 
[49] H. H. Le, M. J. Loomes, R. C. V. Loureiro, Mapping arm movements to robotic sonic interaction promote group dynamics and increase engagement at a task, in: Proceedings of the International Conference on Neurorehabilitation, Vol. 1, Toledo, Spain, 2012, pp. 843-846.

[50] H. Le, R. Loureiro, F. Dussopt, N. Phillips, A. Zivanovic, M. Loomes, A haptically enhanced painting as a tool for neurorehabilitation, in: IEEE International Conference on Rehabilitation Robotics 2013, pp. $24-25$.

[51] H. I. Krebs, B. T. Volpe, M. L. Aisen, N. Hogan, Increasing productivity and quality of care: Robotaided neuro-rehabilitation, Journal of Rehabilitation Research and Development 37 (6) (2000) 639-652.

[52] S. Balasubramanian, J. Klein, E. Burdet, Robot-assisted rehabilitation of hand function, Current Opinion in Neurology 23 (6) (2010) 661-670.

[53] A. Barbosa, E. A. Lamounier Junior, A. O. Andrade, A. Cardoso, Virtual and Augmented Reality: A New Approach to Aid Users of Myoelectric Prostheses, InTech, 2012, Ch. 17, pp. 409-425.

[54] S. Schulz, C. Pylatiuk, M. Reischl, J. Martin, R. Mikut, G. Bretthauer, A hydraulically driven multifunctional prosthetic hand, Robotica 23 (3) (2005) 293-299.

[55] D. Simpson, G. Kenworthy, The design of a complete arm prosthesis, Biomedical Engineering 8 (2) (1973) 56-59.

[56] B. Peerdeman, D. Boere, H. Witteveen, R. Huis in 'tVeld, H. Hermens, S. Stramigioli, H. Rietman, P. Veltink, S. Misra, Myoelectric forearm prostheses: State of the art from a user-centered perspective, The Journal of Rehabilitation Research and Development 48 (6) (2011) 719-737.

[57] J. A. Doringer, N. Hogan, Performance of above elbow body-powered prostheses in visually guided unconstrained motion tasks, IEEE Transactions on Biomedical Engineering 42 (6) (1995) 621-631.

[58] J. Kruit, J. C. Cool, Body-powered hand prosthesis with low operating power for children, Journal of Medical Engineering \& Technology 13 (1-2) (1989) 129-133.

[59] A. Muzumdar, Powered upper limb prostheses: control, implementation and clinical application, Springer, Berlin, Germany, 2003.

[60] P. M. Rossini, S. Micera, A. Benvenuto, J. Carpaneto, G. Cavallo, L. Citi, C. Cipriani, L. Denaro, V. Denaro, G. Di Pino, F. Ferreri, E. Guglielmelli, K. P. Hoffmann, S. Raspopovic, J. Rigosa, L. Rossini, M. Tombini, P. Dario, Double nerve intraneural interface implant on a human amputee for robotic hand control, Clinical Neurophysiology 121 (5) (2010) 777-83.

[61] P. A. Parker, R. N. Scott, Myoeletric prosthesis: State of the art, Journal of Med. Eng. \& Tech. 12 (4) (1988) 143-151.

[62] J. E. Paciga, P. D. Richard, R. N. Scott, Error rate in five-state myoelectric control systems, Med Biol Eng Comput 18 (3) (1980) 287-90.

[63] P. E. Patterson, J. A. Katz, Design and evoluation of a sensory feedback systems that provides grasping pressure in a myoletric hand, Journal of Rehabilitation Research and Development 29 (1) (1992) 1-8.

[64] T. H. Geake, Advanced feedback system for myoelectrically controlled prostheses, Phd thesis (1994).

[65] L. A. Hoff, Errors in frequency parameters of emg power spectra, IEEE transactions on biomedical engineering 38 (11) (1991) 1077-1088.

[66] P. A. O. . SCOTT, E. L. M. R. N., Myoletric signal characteristics from muscles in residual upper limbs, IEEE Trans. Rehab. Eng. 2 (4) (1994) 266-270.

[67] P. Gallagher, D. Desmond, M. MacLachlan, Psychoprosthetics, Springer, London, 2008.

[68] D. J. Atkins, D. C. Y. Heard, W. H. Donovan, Epidemiologic overview of individuals with upper-limb loss and their reported research priorities, Journal of Prosthetics and Orthotics 8 (1) (1996) 2-11.

[69] E. Biddiss, T. Chau, Upper limb prosthesis use and abandonment: A survey of the last 25 years, Prosthetics and Orthotics International 31 (3) (2007) 236-257.

[70] P. J. Kyberd, C. Wartenberg, L. Sandsjö, S. Jönsson, D. Gow, J. Frid, C. Almström, L. Sperling, Survey of upper-extremity prosthesis users in sweden and the united kingdom, Journal of Prosthetics and Orthotics 19 (2) (2007) 55-62.

[71] T. Bertels, T. Kettwig, Breathable liner for transradial prostheses, in: Proceedings of the Myoelectric Controls Symposium (MEC'11), University of New Brunswick, Fredericton, Canada, 2011.

[72] K. Hagberg, E. Häggström, S. Jönsson, B. Rydevik, R. Brånemark, Osseoperception and Osseointe- 
grated Prosthetic Limbs, Springer-Verlag London Limited, London, 2008, Ch. 10, pp. 131-140.

[73] K. Hagberg, R. Brånemark, One hundred patients treated with osseointegrated transfemoral amputation prostheses: Rehabilitation perspective, Journal of Rehabilitation Research \& Development 46 (3) (2009) 331-344.

[74] M. Mori, The uncanny valley, IEEE Robotics and Automation Magazine 7 (2012) 98-100.

[75] P. J. Kyberd, C. Light, P. H. Chappell, J. M. Nightingale, D. Whatley, M. Evans, The design of anthropomorphic prosthetic hands: A study of the southampton hand, Robotica 19 (06) (2001) 593600.

[76] P. J. Kyberd, A. Clawson, B. Jones, The use of underactuation in prosthetic grasping, Mechanical Sciences 2 (1) (2011) 27-32.

[77] P. J. Kyberd, W. Hill, Survey of upper limb prosthesis users in sweden, the united kingdom and canada, Prosthetics and Orthotics International 35 (2) (2011) 234-241.

[78] K. De Laurentis, S. Phillips, Design of a powered two-dof prosthetic wrist, in: Proceedings of the 12th World Congress of the International Society of Prosthetics and Orthotics, 2007, p. 524.

[79] A. Fougner, E. Scheme, A. D. Chan, K. Englehart, O. Stavdahl, Resolving the limb position effect in myoelectric pattern recognition, Transactions on Neural Systems and Rehabilitation Engineering 19 (6) (2011) 644-651.

[80] M. Ortiz-Catalan, R. Branemark, B. Hakansson, J. Delbeke, On the viability of implantable electrodes for the natural control of artificial limbs: review and discussion, Biomedical Engineering Online 11 (2012) 33 .

[81] T. A. Kuiken, G. A. Dumanian, R. D. Lipschutz, L. A. Miller, K. A. Stubblefield, The use of targeted muscle reinnervation for improved myoelectric prosthesis control in a bilateral shoulder disarticulation amputee, Prosthetics and Orthotics International 28 (3) (2004) 245-253.

[82] T. A. Kuiken, L. A. Miller, R. D. Lipschutz, B. A. Lock, K. Stubblefield, P. D. Marasco, P. Zhou, G. A. Dumanian, Targeted reinnervation for enhanced prosthetic arm function in a woman with a proximal amputation: a case study, Lancet 369 (9559) (2007) 371-80.

[83] K. Stubblefield, S. B. Finucane, L. A. Miller, B. A. Lock, Training individuals to use pattern recognition to control an upper limb prosthesis, in: MyoElectric Controls/Powered Prosthetics Symposium (MEC'11 Raising the Standard).

[84] B. Lock, A. M. Simon, K. Stubblefield, L. Hargrove, Prosthesis-guided training for practical use of pattern recognition control of prostheses, in: Proceedings of the Myoelectric Controls Symposium (MEC'11), Fredericton, NB, Canada, 2011.

[85] A. M. Wing, C. Fraser, The contribution of the thumb to reaching movements, The Quarterly Journal of Experimental Psychology Section A 35 (2) (1983) 297-309.

[86] C. Fraser, A. W. Wing, A case study of reaching by a user of a manually-operated artificial hand, Prosthetics and Orthotics International 5 (3) (1981) 151-156.

[87] R. M. Bongers, P. J. Kyberd, H. Bouwsema, L. P. Kenney, D. H. Plettenburg, C. K. Van der Sluis, Bernstein's levels of construction of movements applied to upper limb prosthetics, JPO: Journal of Prosthetics and Orthotics 24 (2) (2012) 67-76.

[88] J. M. Carroll, Scenario-based design. Envisioning work and technology in systems development, 1st Edition, Wiley, New York, NY, 1995.

[89] A. Tapus, M. J. Mataric, B. Scasselati, Socially assistive robotics [grand challenges of robotics], IEEE Robotics and Automation Magazine 14 (1) (2007) 35-42.

[90] W. E. Bijker, T. Hughes, T. Pinch, The social construction of technological systems. New directions in the sociology and history of technology, 7th Edition, MIT Press, Cambridge, Mass, 1999.

[91] J. Weyer, U. Kirchner, L. Riedl, J. Schmidt, Technik, die Gesellschaft schafft. Soziale Netzwerke als Ort der Technikgenese, 1st Edition, Sigma, Berlin, 1997.

[92] G. Abels, A. Bora, Demokratische Technikbewertung, Transcript-Verlag, Bielefeld, Germany, 2004.

[93] A. Strauss, Grundlagen qualitativer Sozialforschung. Datenanalyse und Theoriebildung in der empirischen soziologischen Forschung, 1st Edition, Fink, München, 1994.

[94] J. Friedrichs, Methoden empirischer Sozialforschung, Westdeutscher Verlag, Opladen, 1990. 
[95] W. Rammert, Technik und Innovation, Technical University Technology Studies Working Papers, Technische Universität Berlin, Berlin, 2008.

[96] H. Braun-Thürmann, Innovation, 1st Edition, Transcript-Verlag, Bielefeld, 2005.

[97] S. Frenkel, M. Korczynski, K. Shire, M. Tam, On the front line. Organization of work in the information economy, ILR Press, Ithaca, New York:, 1999.

[98] T. Herrmann, P. Mambrey, K. Shire, Wissensgenese, Wissensteilung und Wissensorganisation in der Arbeitspraxis, 1st Edition, Westdt. Verl, Wiesbaden, 2003.

[99] S. Star, J. Griesemer, Institutional Ecology. Translations and Boundary Objects: Amateurs and Professionals in Berkeley's Museum of Vertebrate Zoology 1907-1939, 1989, pp. 387-420.

[100] M. B. Rosson, J. Carroll, Scenario-based design, 2nd Edition, Erlbaum, Mahwah, NJ, 2003, pp. 10321050.

[101] D. Compagna, Lost in translation? the dilemma of alignment within participatory technology developments, Poiesis \& Praxis 9 (1) (2012) 125-143.

[102] J. Strübing, Symbolischer interaktionismus revisited. konzepte für die wissenschafts- und technikforschung, Zeitschrift für Soziologie 26 (5) (1997) 368-386.

[103] H. Beyer, K. Holtzblatt, Contextual Design. Defining Customer-Centered Systems, Morgan Kaufmann Publishers Inc, San Francisco, California, 1998.

[104] A. Cockburn, Writing Effective Use Cases, Cockburn, Highsmiths Series Editors. Addison-Wesley, 2002.

[105] ISO, ISO 9241-210:2010 ergonomics of human-system interaction - part 210: Human-centred design for interactive systems (2010).

[106] A. Cooper, The Inmates Are Running The Asylum, Sams, 1999.

[107] D. Compagna, S. Derpmann, K. Mauz, The operation of autonomous mobile robot assistants in the environment of care facilities adopting a user-centered development design, Enterprise and Work Innovation Studies 5 (5) (2009) $11-24$.

[108] S. Walter, K. Limbrecht, C. S., V. Hrabal, H. Traue, The individual Adaption Module (iAM): A framework for individualization and calibration of companion technologies, Taylor \& Francis, CRC Press, 2012, pp. 614-623.

[109] S. Walter, J. Kim, D. Hrabal, S. Crawcour, H. Kessler, H. Traue, Transsituational individual-specific biopsychological classification of emotions, IEEE Transactions on Systems, Man, And Cybernectics Part A: Systems and Humans.

[110] S. Biundo, A. Wendemuth, Von kognitiven technischen systemen zu companion-systemen, Künstliche Intelligenz 24 (4) (2010) 335-339.

[111] M. Bonfe, F. Boriero, R. Dodi, P. Fiorini, A. Morandi, R. Muradore, L. Pasquale, A. Sanna, C. Secchi, Towards automated surgical robotics: A requirements engineering approach, in: Proceedings of the 4th IEEE RAS and EMBS International Conference on Biomedical Robotics and Biomechatronics (BioRob), 2012, pp. 56-61. 